Provided by the author(s) and University of Galway in accordance with publisher policies. Please cite the published version when available.

\begin{tabular}{|c|l|}
\hline Title & $\begin{array}{l}\text { Architecture for a Home Media Network Based on PTP-IP } \\
\text { Connectivity Techniques }\end{array}$ \\
\hline Author(s) & Corcoran, Peter \\
\hline $\begin{array}{c}\text { Publication } \\
\text { Date }\end{array}$ & 2008-08-05 \\
\hline $\begin{array}{c}\text { Publication } \\
\text { Information }\end{array}$ & $\begin{array}{l}\text { Corcoran, P. (2008). Architecture for a Home Media Network } \\
\text { Based on PTP-IP Connectivity Techniques. Paper presented at } \\
\text { Technical Papers. }\end{array}$ \\
\hline $\begin{array}{c}\text { Publisher } \\
\text { IEEE }\end{array}$ \\
\hline $\begin{array}{c}\text { Link to } \\
\text { publisher's } \\
\text { version }\end{array}$ & $\begin{array}{l}\text { http://www.ieeexplore.ieee.org/search/srchabstract.jsp?tp=\&arn } \\
\text { umber=4588006 }\end{array}$ \\
\hline $\begin{array}{c}\text { Item record } \\
\text { http://hdl.handle.net/10379/1343 }\end{array}$ \\
\hline
\end{tabular}

Downloaded 2023-04-26T08:04:37Z

Some rights reserved. For more information, please see the item record link above. 


\title{
Architecture for a Home Media Network Based on PTP-IP Connectivity Techniques
}

\author{
Peter Corcoran, Member, IEEE \\ National University of Ireland, Galway
}

\begin{abstract}
PTP/IP functionality is extended to enable networked devices to be organized in a sequential media processing workflow. The additional device functionality necessary to support this network architecture is not unduly complex and the workflow organization can be readily described through an intuitive user interface. This research offers an interesting alternative to conventional multimedia networking.
\end{abstract}

\section{I.INTRODUCTION}

Picture Transfer Protocol (PTP) is a transport and platform independent standard [1] that enables communication and data exchange between digital cameras and other devices with USB interfaces [2]. With PTP [5-6], digital cameras can exchange images with host computers, printers, and other digital capture and display devices. PTP is also the basis for Media Transfer Protocol (MTP) [8] which extends the functionality of PTP to manage content on portable media players.

An extended standard to provide a TCP/IP compatible transport for PTP became a CIPA standard in 2005 [3, 4]. It enables PTP, which was previously restricted to peer-to-peer USB device connections, to be used with TCP/IP networks. Multiple simultaneous connections among digital imaging devices, which was not possible with PTP over USB, is supported in this PTP-IP standard. In earlier work [8] we described network appliances and their protocol stacks to enable legacy PTP devices to interact with PTP-IP devices on a TCP/IP network. In this paper we consider how PTP-IP functionality could be further extended to enable networked devices to be organized in a sequential media processing workflow. This approach offers an interesting alternative to more conventional approaches to home multimedia networking.

\section{Device Types}

Data or Media storage devices (DSD/MSDs) are PTP devices whose function is limited to storing and managing media content and to providing access to such content over a network connection. Examples include a basic PTP digital camera, PTP-enabled network storage appliances, memory card readers and networked appliances containing optical or magnetic disc storage. In essence, any device with stores digital content and is attached to a network can be enabled with a PTP-IP adapter [8] to act as a DSD/MSD.

Functional server devices (FSDs) are more sophisticated devices which can process digital content. Such devices can support enhancements to standard PTP networking. Each FSD contains software components to implement (i) PTP Link Routing; (ii) User Interface support logic and (iii) PTP connection management. Typically such a device will determine a list of other network devices and which of these it should connect to. Normally this will be decided based on the media types that the FSD can handle.

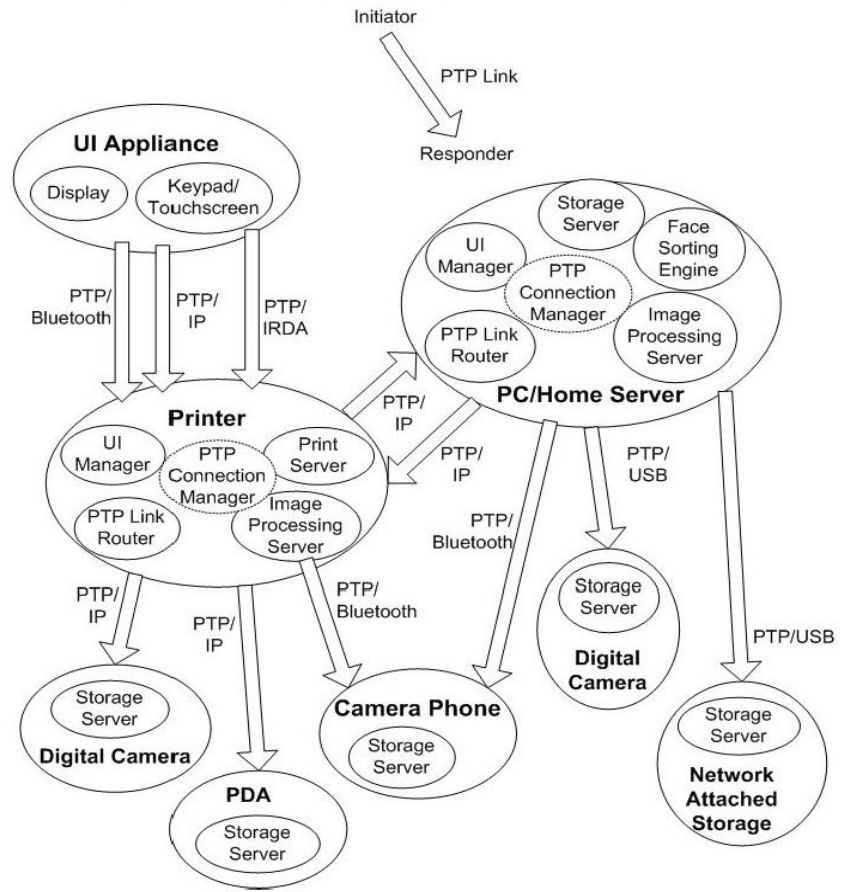

Fig 1: Interconnected devices in our PTP-IP home network architecture; note how Bluetooth, TCP/IP and $I R$ devices may potentially interoperate.

\section{Components of AN FSD}

\section{A. PTP Connection Manager Module}

This software component performs the function of determining which network devices to connect to and is also responsible for managing PTP connects and disconnects, and maintaining existing PTP links. 


\section{B. PTP Link Router (PTP-LR)}

The function of the PTP Link Router (PTP-LR) is to route PTP commands, data and events between a device on which the PTP-LR is running and other connected PTP devices. Typically a PTP link router will respond to a single PTP connection established by another PTP Device which acts as a primary initiator and sets up a routing table for that connection.

Where any one of the connections is to another FSD, the PTP-LR on the current device makes an enquiry of the instance of PTP-LR on the other FSD to determine established connections. This recursion continues until the last PTP-LR in a chain has no connected FSD's to check. NonFSD devices which are added to this PTP link chain can only act as responders to their local Initiator and thus will terminate that branch of the PTP link chain in a natural manner.

\section{User Interface Description Manager Module}

This module is active on any FSD which can act as a Secondary Initiator. It is used as local data store of device description information from PTP devices which are connected to the current device. An example implementation of the UI which is suitable for a PDA or smartphone device is illustrated in Fig 2 below.

\section{REFERENCES}

1. ISO 15740, "Picture transfer protocol (PTP) for digital still photography devices", Photography - Electronic still picture imaging, 2005

2. USB Implementer's Forum, "Universal Serial BusStill Image Capture Device Definition Revision 1.0", htto: / w w w usb. org/developersidevclass docsin sb still im g10.pdf July 2000.

3. Camera \& Imaging Products Association (CIPA), "PTP-IP Picture Transfer Protocol over IP version 1.0", http://www.cipa.jp/hyoujunka/kikaku/pdf/DC-X005.pdf, Japan, 2005.

4. P. Bigioi, G. Susanu, E. Steinberg, and P. Corcoran, "PTP/P - a new transport specification for wireless photography", Consumer Electronics, IEEE Transactions, vol. 51, Issue 1, pp. 240-244, Feb. 2005.

5. P. Bigioi, G. Susanu, P. Concoran, and I. Mocanu, "Digital camera connectivity solutions using the picture transfer protocol (PTP)", Consumer Electronics, IEEE Transactions vol. 48, Issue 3, pp. $417-427$, Aug. 2002.

6. P. Bigioi, P. Corcoran, and G. Susanu, "Digital imaging services using PTP (Picture Transfer Protocol)", Consumer Electronics, 2002. ICCE. 2002 Digest of Technical Papers, pp. 54-55, June 2002.

7. Microsoft, "MTP Media Transfer Protocol Specification", http://msdn.microsoft.com/library/default.asp?url=/ibrar Ven-us/dnwmt/html/mtp spec.asp

8. P. Bigioi, G. Susanu, E. Steinberg, and P. Corcoran, "" PTP/IP Adapter Design and Connectivity Techniques for Legacy Imaging Appliances "", Consumer Electronics, IEEE Transactions, in press May 2007.

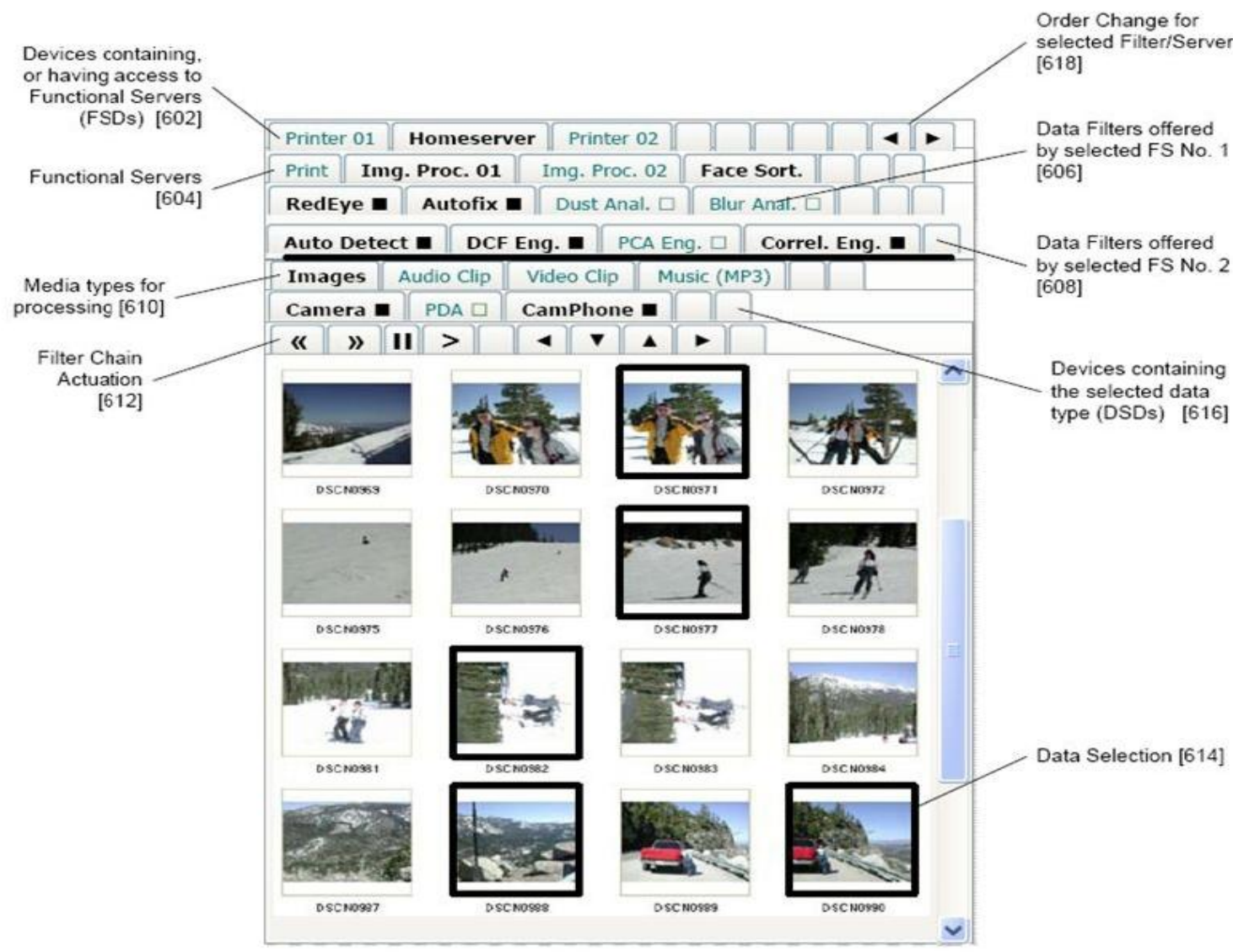

Fig 2: User Interface derived from the connected architecture of Fig 1. 